\title{
DISCUSSION AFTER SESSION C
}

Douglas: With reference to selecting radio source counterparts for optical astrometric measurement, angular structure enters in two ways:

(1) If angular structure is large compared to the scale of interferometer fringes, the radio centroid is not measurable; such sources should not be chosen.

(2) Finite angular structure which is small compared to interferometer fringes will not impede measurement of radio brightness centroid. However, it may alter the confidence with which one assumes radio and optical centroids to be coincident. In fact, for an individual source, such an assumption is never fully safe, so radio-optical comparisons should be statistical.

Elsmore: I agree with Dr Douglas but I would add that sometimes the optical object is extended : in particular, 3C 48 has a jet to the north of this object, as shown in plates by Sandage. It is interesting to note that Dr Wade showed that both the NRAO and Cambridge radio positions lie north of the optical centroid.

Moffet: As a partial answer to Mr Murray's question (as to which radio sources have compact components), we have found that essentially all sources with pronounced centimeter-excess radio spectra have components of the order of 0 ".001 diam. In the absence of convincing evidence to the contrary, we assume that the compact radio and optical components of such objects are coincident. At longer wavelengths the centroids of the radio sources may be displaced because asymmetric large-scale components are also present, as is the case for 3C279. Mr Elsmore has already mentioned that extended components may similarly affect optical positions. Nevertheless it is important to have good optical positions for the compact components of even very complex objects such as the nuclei of Seyfert galaxies (for example NGC 1275 and 3C 120).

Tucker: Does intergalactic dispersion separate the positions of the radio source and the optical counterpart?

Wade: There is no evidence of such dispersive shifts to the 0 ". 1 level, although it has been sought. 